\title{
Interacciones étnicas e indicadores de desigualdad social en el Cementerio de La Falda (SJTil 43), Tilcara, Jujuy ${ }^{1}$
}

María de LA AsUnCIÓn BORDACH²

\section{RESUMEN}

Se estudian comportamientos funerarios en el Cementerio de La Falda (sitio SJTil 43) considerando que: a) las estructuras funerarias encierran una porción del comportamiento funerario; $b$ ) diferentes identidades sociales pueden ser inferidas a través de la cantidad y calidad de los itemes materiales y no materiales; c) en sociedades andinas es factible explorar aspectos ligados al género, parentesco, y posición social que se distinguen por su profundo significado y simbolismo, cosmovisión e ideología. El análisis es arqueológico y etnohistórico. Se observa que el cementerio de La Falda muestra una separación bien marcada en el rol social por sexo. Puesto que el análisis de las inclusiones funerarias sugiere un estatus social alto, se concluye que se trataría de un grupo integrado por miembros de estados vecinos y con estatus oficial equivalente a "incas de privilegio".

Palabras claves: arqueología funeraria - complejidad social - Inca-Hispanoindígena - Noroeste Argentino.

\begin{abstract}
This paper focuses on the study of funerary behavior in the La Falda burial site (site SJTil 43), to consider that: a) Funerary structures enclose aspects of funerary behavior; b) Different social identities can be inferred by studying the quantity and quality of material items; c) In Andean societies, it is possible to explore aspects linked to gender, kinship, and social status, made distinct by their profound meaning, symbolism, and ideology. Analysis is both archaeological and ethnohistorical. At La Falda, a separation of social roles by gender is clearly observed. Because the analysis of funerary inclusions suggests an adscription to a high social status, it is concluded that the individuals buried at the site could well belong to a group formed by members coming from bordering states, with an official status equivalent to that of the "incas de privilegio".
\end{abstract}

Key words: funerary archaeology - social complexity Inca-Early Spanish Contact - Northwestern Argentina.

Recibido: septiembre 2005. Aceptado: febrero 2006.

1 Trabajo realizado con fondos otorgados por la Agencia Nacional de Promoción Científica y Tecnológica; Secretaría de Ciencia y Técnica, Universidad Nacional de Río Cuarto, Fundación Antorchas, Agencia Córdoba Ciencia, Sociedad del Estado; CONICET.

\section{Introducción}

En toda sociedad humana, el fenómeno de la muerte suele ocasionar el despliegue de una variedad de comportamientos cuya naturaleza es tanto colectiva como individual. Sin entrar a considerar el mayor o menor grado de espontaneidad emocionalmente ligada a la materialización de la expresión de los mismos, en general, tales respuestas o reacciones son puestas de manifiesto y/o canalizadas mediante la observación de un conjunto de pautas socialmente especificadas. El escenario donde se verifican el ritual de la muerte y su programa mortuorio comúnmente involucra dos tipos de actores principales: el muerto (o los muertos) y sus deudos. Tanto el desarrollo de este ritual como el de su programa asociado están destinados a posibilitar un tránsito socialmente elaborado que vuelve factible para ambas partes concretar la materialización del abandono del mundo de los vivos y el ingreso al mundo de los muertos. Entre una multiplicidad de aspectos, y salvo situaciones de excepcionalidad, por lo general suelen desplegarse en este escenario los roles y el conjunto de las identidades sociales de los individuos participantes (Linton 1936; Goodenough 1965; Saxe 1970; Brown 1971; Tainter 1978; Pader 1982). En aquellas sociedades que no incineran o que no se desprenden materialmente de los cuerpos de sus muertos, es posible verificar que, por lo menos, una porción de todo el despliegue de tales comportamientos socialmente pautados y que hacen al ritual funerario queda incorporada a la estructura que habrá de contener los restos inhumados. Cualquiera sea su nivel de complejidad y la cantidad de energía invertida en su confección, en ella quedará guardado el testimonio, aunque muchas veces tan solo un vesti-

\footnotetext{
2 Laboratorio de Osteología y Anatomía Funcional Humana, Depto. de Ciencias Naturales, Facultad de Ciencias Exactas, Físico-Químicas y Naturales, Universidad Nacional de Río Cuarto, Agencia Postal No 3. X5804BYA. Email: mbordach@exa.unrc.edu.ar
} 
gio, de aquellas pautas sociales que han condicionado la intencionalidad de los deudos, últimos responsables por los modos o las formas en que se materializan tanto el rito como las inclusiones que acompañen al difunto. Es esta circunstancia la que hace posible la existencia de las fuentes críticas de información contextual necesarias para proceder al estudio de lo que se ha dado en denominar la "arqueología de la muerte" (sensu Chapman y Randsborg 1981). Esta es la razón por la cual, en todas las sociedades humanas, y muy particularmente en aquellas involucradas en el surgimiento y en el desarrollo de la complejidad, los sitios de enterratorio colectivo constituyen una fuente potencial de información para explorar, a través de los indicadores arqueológicos del comportamiento mortuorio, configuraciones posibles de la sociedad que los ha generado (Chapman 1977; Hodder 1982; Härke 1997; Parker Pearson 2000).

De este modo, el análisis de las prácticas funerarias no se agota en la simple descripción de los rasgos, relaciones espaciales, ni en su determinación cronológica, sino que se estima que puede, además, proporcionar una multiplicidad de testimonios vinculados con el parentesco, el género y la posición social (Parker Pearson 2000). Estos testimonios generalmente se distinguen por su profundo significado y simbolismo, y pueden utilizarse para determinar la presencia de personas sociales y sus estatus posibles, así como también intentar exploraciones vinculadas con la cosmovisión y la ideología (Pader 1982; Parker Pearson 1982; Härke 1997). Por todas estas circunstancias el programa mortuorio ha sido definido como el conjunto coherente y estructurado de comportamientos sociales que organiza lo que colectivamente se considera una disposición apropiada de los muertos (Brown 1971). Puesto que la presencia de evidencia material puede terminar siendo el resultado de una intencionalidad de los deudos y no necesariamente un referente directo de la real configuración social del difunto, el estudio del comportamiento mortuorio debe comprender el análisis de todos los elementos disponibles que puedan llegar a formar parte de la ceremonia funeraria $\mathrm{y}$, por ende, incluir tanto los referentes visibles como los no visibles (Chapman y Randsborg 1981; Hodder 1982; Moore y Scott 1997). Este tipo de análisis vuelve factible, cuando es adecuadamente aplicado en estudios arqueológicos: a) poner de manifiesto las interrelaciones entre las identidades de las personas, las redes y las reglas de interacción social; b) evidenciar los modos de empleo de la cultura material en las prácticas mortuorias (Chesson 2001); c) observar de qué manera lo ritual-ceremonial se materializa en el contexto arqueológico, y d) de qué modo la memoria de los ancestros se expresa en la vida política e ideológica (Kaulicke 1997).

La excavación sistemática del sitio SJTil 43, también conocido como Cementerio de La Falda (Figura 1) proporciona una excelente oportunidad para aplicar el conjunto de conocimientos que conforman el marco teórico referido, e intentar una comprensión del significado de la información recuperada (Bordach et al. 1998; Grosso et al. 1998, 2001-2002; Mendonça et al. 1997, 2003; Bordach y Mendonça 2001; Mendonça y Bordach 2001). El Cementerio de La Falda constituye un sitio de enterratorio colectivo objeto de excavaciones sistemáticas durante el desarrollo de nuestras investigaciones en la quebrada de Humahuaca (localidad de Tilcara, Prov. de Jujuy) en la década de 1990. La importancia del Cementerio de La Falda reside en que representa un momento poco conocido de la historia regional (Mendonça et al. 1997). Por sus asociaciones culturales, este cementerio fue inicialmente ubicado en el siglo XVI. Varios fechados radiocarbónicos permitieron precisar su correspondencia temporal entre 1535 y 1595 DC (Mendonça y Bordach 2001). En la actualidad, y como consecuencia de una expansión constructiva de los últimos años el sector donde se hallaba este cementerio (Mendonça et al. 1997; Bordach et al. 1998; Bordach y Mendonça 2001) ha quedado cubierto por la expansión edilicia que sufre el actual tejido urbano del pueblo de Tilcara.

Entre otros aspectos, en este cementerio se verifica una notable segregación espacial respecto de todo otro indicio de ocupación humana; las características estructurales de las unidades inhumatorias responden a un patrón no conocido y mucho menos generalizado en la región; el estado de preservación de las estructuras inhumatorias y de los materiales intracámara es excepcional; sus inclusiones funerarias que son tanto locales como de influencia incaica, y también europea (Mendonça et al. 1997; Bordach y Mendonça 2001); y la naturaleza y disposición espacial, genérica, y simbólica de cuerpos e inclusiones funerarias ameritan un tratamiento detallado (Barboza et al. 2001, 2002a, 2002b y 2003-04). 


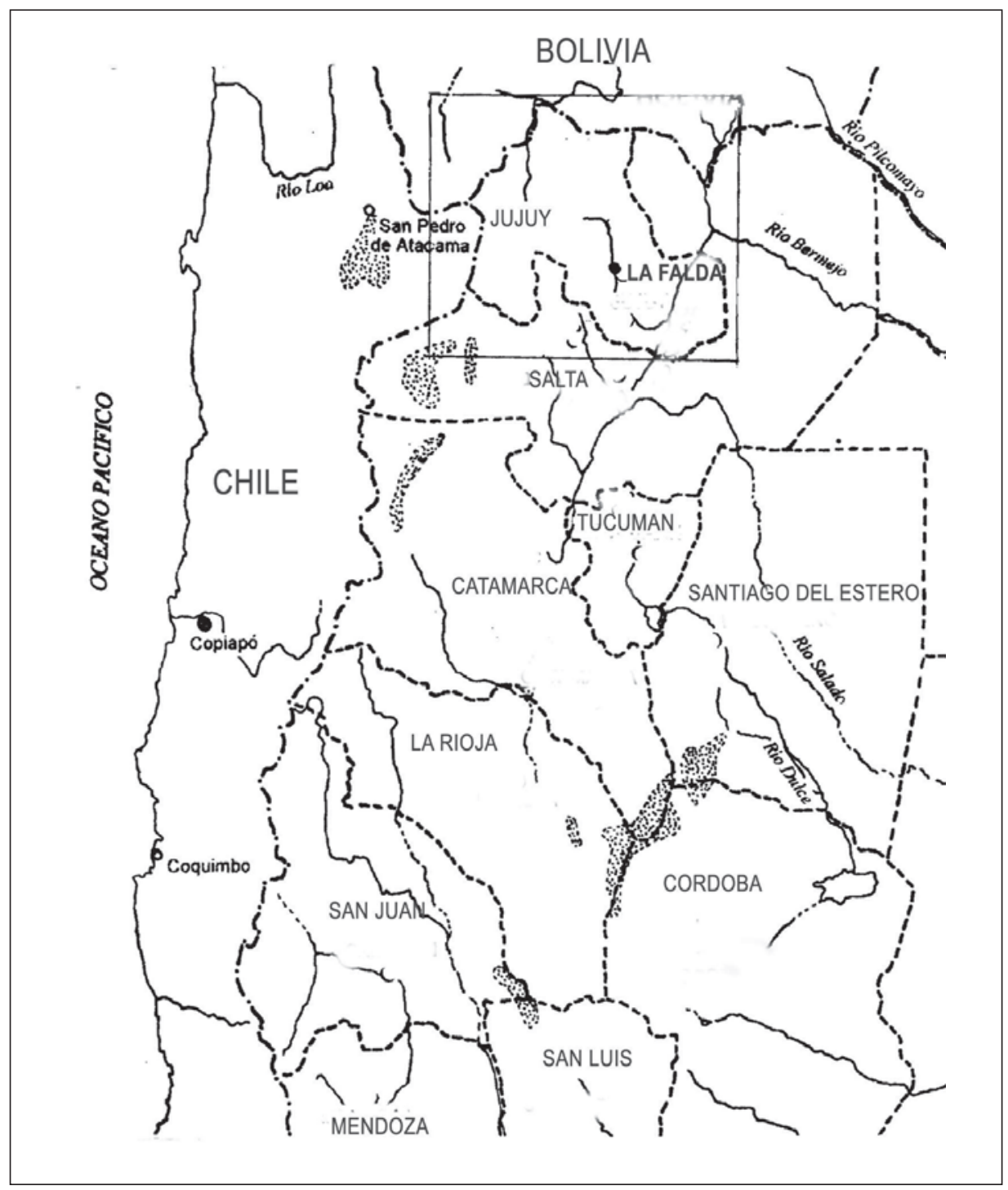

Figura 1. Mapa del Noroeste Argentino y países limítrofes que muestra la ubicación del sitio SJTil 43 (Cementerio La Falda) en Tilcara, quebrada de Humahuaca, Provincia de Jujuy.

Por todo ello, en este trabajo intentaré explorar el valor potencial de la evidencia funeraria tanto material como no material del comportamiento mortuorio del grupo humano que generó esta área de enterratorio colectivo. Para ello recurriré al análisis comparativo y crítico de la información recopilada por tumba y por conjuntos de tumbas, considerándolos potenciales indicadores de la existencia de posibles desigualdades sociales. Dado que para el lapso temporal considerado se cuenta con datos de carácter etnohistórico, el análisis arqueológico será complementado con las fuentes documentales escritas disponibles.

\section{Materiales}

Conforme al corpus de conocimientos arqueológicos actualmente disponibles, las tumbas de $\mathrm{La}$ Falda efectivamente constituyen hallazgos excepcionales en la quebrada de Humahuaca. Fueron excavadas en los sedimentos de las laderas de una de las serranías secundarias del Cordón de Zenta que enmarcan por el este al conoide tilcareño. Todas las tumbas, salvo una, se pueden encuadrar dentro de la tipología de cámaras sepulcrales genéricamente denominadas "pozo con cámara lateral” (Krapovickas 1977; Tarragó 1984). Se las 
conoce también como "shaft tombs" o tumbas en forma de bota o "botiformes". En La Falda, están excavadas en una secuencia estratigráfica bien definida de sedimentos, donde se alternan capas limosas, arcillosas y arenosas. Una vez que se encontraba un estrato arenoso de adecuada potencia, a una profundidad que oscila entre 1 y $2 \mathrm{~m}$, se procedía a la excavación del receptáculo funerario propiamente dicho. Para ello, se conformaba una suerte de ampolla lateral de la "chimenea" o diáfisis de la tumba. Por lo general, esta ampolla es muy espaciosa y en ella se depositaban el o los cadáveres con sus respectivas inclusiones funerarias. Una vez terminado el acto de inclusión funeraria, un tabique de lajas superpuestas fijadas con barro batido separaba la cámara funeraria (bóveda) propiamente dicha, del fuste o "chimenea" que posibilitaba el ingreso desde la superficie del terreno. Es común hallar en la chimenea, junto a las lajas, restos de ofrendas de quema (generalmente una olla con restos de material carbonizado en su interior). Finalmente, el tubo de ingreso era tapado con sedimentos de manera tal, que el acceso a la tumba quedaba cerrado por una pila subcilíndrica de relleno intencional considerablemente alta. Este último acto determinaba que la estructura funeraria quedara absolutamente no visible en superficie (Mendonça et al. 1997; Bordach y Mendonça 2001).

En este cementerio el tratamiento normativo parece ser el entierro primario e individual, siendo poco frecuente el hallazgo de una doble inhumación (Bordach et al. 1998) Cuando esta circunstancia se verifica, se trata -en todos los casos por nosotros observados-, de individuos del mismo sexo. Los cuerpos están dispuestos en su mayoría en posición decúbito lateral o dorsal y están siempre fuertemente flexionados. Cuentan con su indumentaria, objetos personales y demás inclusiones (Figura 2). Como ya hemos mencionado, en estos enterratorios se da la presencia de inclusiones funerarias locales con y sin influencia incaica, así como también materiales de origen europeo, todo ello en un contexto mortuorio (forma de la tumba, posición del cuerpo, inclusión de ofrendas) que responde a un patrón netamente aborigen (Mendonça et al. 1997; Bordach et al. 1998).

En este cementerio se han detectado 25 rasgos funerarios, de los cuales 11 se encontraban intactos. Dos eran rasgos expuestos accidentalmente por la ampliación de una calle, y 12 son recupe- raciones de saqueos realizados en tiempos subactuales. De las 11 tumbas intactas, una de ellas, la $n^{\circ} 3$ constituye una excepción a la regla constructiva en forma de bota (Figura 3). Se tra-

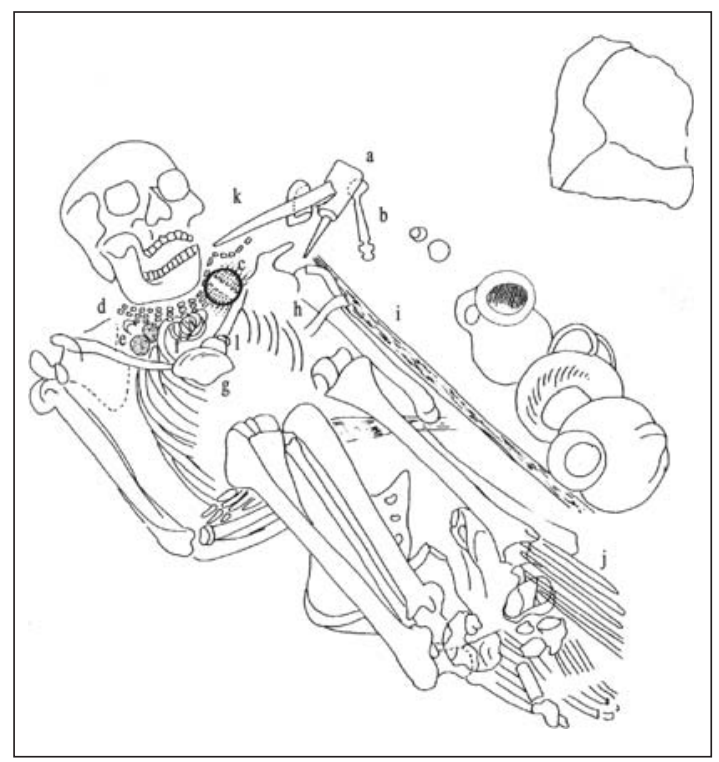

Figura 2. SJTil 43, Rasgo 21. Enterratorio masculino en tumba botiforme que presenta numerosas inclusiones.

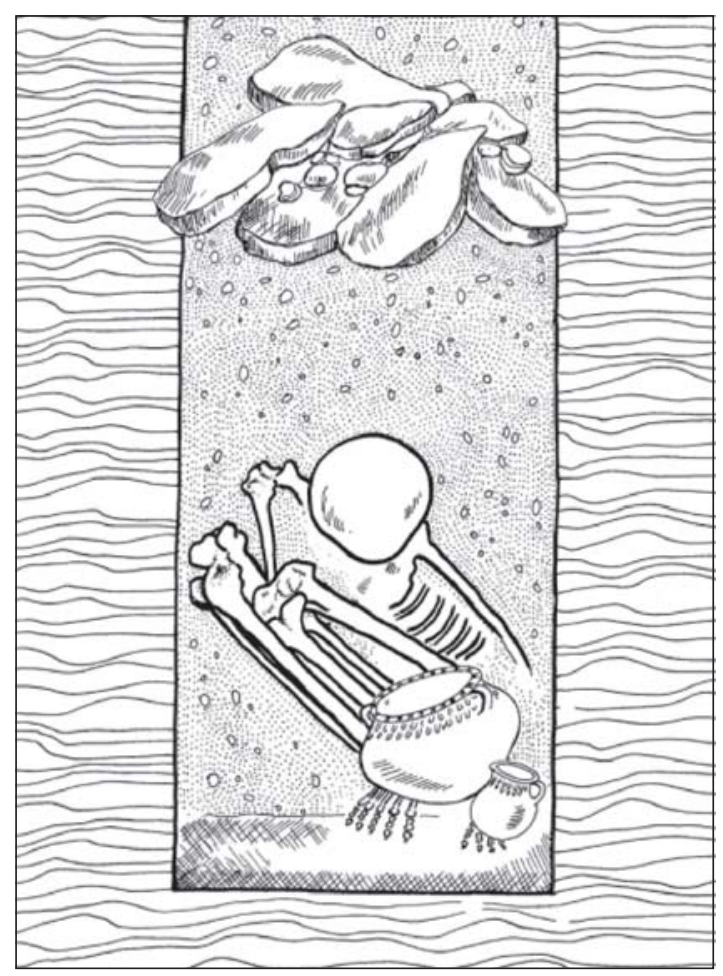

Figura 3. SJTil 43 Rasgo 3. Enterratorio femenino. Tumba excavada en "fuste", planta subcilíndrica, con inclusiones escasas. 
ta, en efecto, de una tumba excavada en fuste o "chimenea" de planta subcilíndrica, pero que carece de su cámara en bóveda lateral. En el fondo de este fuste o "chimenea", a $1.50 \mathrm{~m}$ de profundidad, se encontró el esqueleto de una mujer depositada en posición sedente, con dos ollas de estilo Angosto Chico Inciso y un fragmento de ocre a sus pies (Mendonça et al. 1997). Un conjunto de lajas superpuestas a modo de cierre en falsa bóveda coronaba a modo de tapa el techo de esta tumba. La disposición no en decúbito del cuerpo, sino en forma sedente, y las características de las inclusiones, que no denotan influencias estilísticas altoandinas ni culturales españolas, son también atributos exclusivos de este enterratorio.

Otras inclusiones indistintamente colocadas respecto del sexo comprenden macrorrestos vegetales y animales, calabazas y keros de madera. Se han hallado también objetos de origen europeo, tales como cuentas de vidrio venecianas, fragmentos muy oxidados de hierro, semillas de uva. El individuo de la tumba 17 estaba envuelto en una tela rústica (awaska). Parte de su vestimenta estaba confeccionada en una tela muy fina hecha en lana de vicuña o alpaca, que probablemente constituya un tejido cumbi. Presentaba, además, en su indumentaria, un fragmento de lo que podría ser una camisa de fina batista española. En este fragmento de batista se pudieron identificar tres ojales perfectamente dispuestos a modo de pasacinta. También se encontraron varios trozos de lo que parece haber sido una chaqueta de terciopelo de color marrón habano (Mendonça et al. 1997; Mendonça y Bordach 2001; Bordach et al. 1998; Bordach y Mendonça 2001).

Los individuos recuperados en La Falda, se disponen según las estructuras descritas en la Tabla 1.

\section{Consideraciones teóricas y prácticas}

Uno de los principales objetivos en el estudio de la cultura material asociada a los enterratorios es la búsqueda de regularidades que permitan detectar aspectos vinculados con la persona social del inhumado. Intimamente relacionada con esta circunstancia se encuentra la determinación arqueológicamente observable de los múltiples roles que pudieron haber contribuido a la configuración de dicha persona social (Parker Pearson 2000). Estas identidades constituyen el estatus, el cual a su vez se encuentra en la base estructural de la desigualdad social (Maurer Trinkaus 1995). En términos generales, las distintas identidades sociales pueden inferirse a partir de la cantidad y calidad de los objetos de cultura material que acompañan al muerto, y que forman parte del patrón funerario. Pero los deudos son los protagonistas activos de la ceremonia funeraria (Hodder 1982; Parker Pearson 1982), y por razones políticas, sociales, religiosas o económicas también socialmente pautadas pueden, bajo ciertas circunstancias, anteponer sus intenciones en la representación material del ritual funerario. Advertidos respecto de una lectura simplista de la información recuperada, así como de una interpretación unilineal y directa de los datos obtenidos en las excavaciones de áreas de enterratorio, nos atendremos, no obstante, en nuestro análisis a las formulaciones de Arriaga (1621), Cieza de León, (1922 [1553]) y Silverblatt (1987), quienes consideran que en las sociedades andinas las representaciones funerarias se encontrarían libres de tales sesgos de intencionalidad (Silverblatt 1987).

Tal como suele darse en muchos enterratorios andinos, los individuos de La Falda fueron inhumados llevándose con ellos si no todas, al menos muchas de sus pertenencias. Esto comprende inclusiones tanto incidentales como intencionales (O'Shea 1984), ya que se recuperaron en las excavaciones elementos personales, vestimentas, objetos de adorno, útiles e instrumentos (Silverblatt 1987). También se encuentran ofrendas. Se trata de inclusiones proporcionadas exclusivamente por los deudos y que suelen consistir en alimentos, bebidas y sus recipientes contenedores, entre otros.

Aunque es un hallazgo excepcional en quebrada de Humahuaca, el tipo de tumba "botiforme" ha sido encontrado en otros lugares del Noroeste Argentino y también en países vecinos. Por ejemplo, Krapovickas (1977), describe una tumba de cámara lateral en Cerro Colorado, en la puna; en Cachi Adentro, Tarragó (1984) ha encontrado tumbas de este tipo, con un contexto de claros elementos de origen europeo $\mathrm{y}$, al parecer, siempre vinculadas a la dominación incaica. También se las halló en los alrededores de Santiago (Chile) (M. Uribe, com. pers. 2004), y en Copiapó (Niemeyer 1986). Si bien la extensión espacial de estas estructuras es muy amplia en la región andina, se observa que tan sólo algunos grupos enterraban a sus muertos en shaft tombs (Cadavid 


\begin{tabular}{|c|c|c|c|}
\hline Rasgo & Femeninos & Masculinos & Inclusiones \\
\hline 3 & Maduro/Senil & & Dos ollas Angosto Chico Inciso, ocre. \\
\hline 9 & Subadulto & & $\begin{array}{l}\text { Aribaloide, pucos, plato, olla, palo cavador, ocre, anillo metal, tupus bronce, cuentas } \\
\text { venecianas y vegetales, torteros madera, huesos de camélido }\end{array}$ \\
\hline 18 & Maduro (2) & & $\begin{array}{l}\text { Aribaloides, jarras c/asa, olla, pucos, ruqui }{ }^{2} \text {, torteros, vincha de metal, cestería, } \\
\text { cuentas venecianas, tupus de bronce y plata, punta y keros madera, alisador, calabaza } \\
\text { c/palitos, hierro, palitos. }\end{array}$ \\
\hline 19 & Adulto & & $\begin{array}{l}\text { Aribaloide, olla asa labio adherida, ruqui, ocre, calabaza, keros, bandeja y cuchara } \\
\text { madera, torteros madera y cerámica, tupus y anillos bronce y plata, vincha de plata, } \\
\text { cuentas venecianas, ave. }\end{array}$ \\
\hline 25 & Subadulto & & $\begin{array}{l}\text { Aribaloide, olla globular, puco, pushka, cuchara y lanzadera madera, calabaza, } \\
\text { metapodio de camélido, torteros hueso, topus de plata, cestería, cuentas venecianas } \\
\text { y turquesa, restos ave. }\end{array}$ \\
\hline 11 & & Adulto & $\begin{array}{l}\text { Vasija (con ave), platos, cincel bronce, puntas de proyectil de hueso, pulidor, me- } \\
\text { tal (escoria). }\end{array}$ \\
\hline 16 & & Adulto & $\begin{array}{l}\text { Cincel c/funda de cuero, cincel enmangado, keros, arco, vaso, cuchara y astiles ma- } \\
\text { dera, calabaza, puntas de proyectil de hueso, huesos de camélido, piedra de afilar, } \\
\text { bolsitas con pigmento. }\end{array}$ \\
\hline 17 & & Adulto & $\begin{array}{l}\text { Vasijas, aribaloide, pucos, arco de madera decorado, cuchara y puntas de proyectil } \\
\text { de madera, peine y agujas de espinas de cardón, calabazas, cincel de bronce } \\
\text { enmangado, vincha de plata, cascabel y pinza depilatoria de bronce, tubo insuflatorio, } \\
\text { hueso c/corteza, bolsita c/pigmento, huesos de camélido, chuspa, campana de bron- } \\
\text { ce y hierro, tocado de lana, tejidos awasca y cumbi, batista c/ojales, terciopelo. }\end{array}$ \\
\hline 20 & & Adulto & $\begin{array}{l}\text { Olla, pucos, } \text { virque }^{3} \text {, cincel, placas de bronce, tableta de madera, llijta, cordel, madera } \\
\text { con muesca y huellas de tiras de cuero, puntas de proyectil de hueso, bolsitas c/ pig- } \\
\text { mento, tubo insuflatorio, dos patas de ave, y cuatro marlos de maíz tapados por un } \\
\text { puco, cuerdita de fibra vegetal anudada a una de las patas, punzón de bronce enmangado. }\end{array}$ \\
\hline 21 & & Maduro & $\begin{array}{l}\text { Jarra, puco, cántaro, disco y cascabeles de bronce, cincel y punzón de bronce } \\
\text { enmangados, cuentas venecianas, peine, cuchara y arco madera, llijta, brazalete, } \\
\text { cinta y anillo de plata, calabazas, astiles, valva de molusco, bolsas c/pigmentos. }\end{array}$ \\
\hline 23 & & Subadulto & $\begin{array}{l}\text { Olla, jarra, puco, calabaza, cincel de hierro enmangado, lámina, hachuela, cincel y bra- } \\
\text { zalete de bronce, cuentas venecianas, astiles, tubo insuflatorio, bolsitas c/pigmento, } \\
\text { calabaza, hemidiáfisis de camélido, pala de madera, tela decorado con damero pe- } \\
\text { queño }(3-4 \mathrm{~mm}) \text {. }\end{array}$ \\
\hline
\end{tabular}

Tabla 1. Tumbas con inhumaciones masculinas y femeninas. En negrita se destacan las inclusiones consideradas asociadas al sexo.

Camargo y Ordóñez 1989; Goldstein 1989; Drennan 1995; Duque Gómez 1999). Si bien se trata de rasgos no visibles desde la superficie, las tumbas botiformes de La Falda (excavación del fuste, excavación de la cámara, inhumación e inclusiones, sellado de la bóveda con lajas y argamasa, ofrendas votivas, y finalmente relleno del fuste) se caracterizan por una excepcional inversión de energía en su construcción. En ellas se destaca notablemente la importancia de la verti-

3 Término utilizado en el Cuzco para designar a un metapodio de llama o vicuña, liso y con un extremo aguzado que se emplea para apretar la trama del tejido.

4 Olla grande utilizada para almacenar chicha. calidad, aunque esta sea negativa, inversa. En este sentido, constituyen obras monumentales (Doyon 2002).

Como fuera mencionado, en el sitio se ha hallado una sola excepción a este complejo procedimiento constructivo. Se trata del entierro de una mujer de edad avanzada en un pozo subcilíndrico de 0.80 $\mathrm{m}$ de diámetro y $1.5 \mathrm{~m}$ de profundidad, cuyo cierre estaba coronado por una suerte de tapa circular formada por lajas imbricadas (cámara 3). Como puede observarse en la Figura 3, la localización de este rasgo no permite excluirlo espacialmente de los límites propios de este cementerio. Por lo tanto, consideramos que no se trata de un hallazgo intrusivo, sino que, pese a su relativa atipicidad, 
efectivamente pertenece al conjunto funerario de La Falda. Su sexo y edad, las escasas inclusiones intencionales, la ausencia de inclusiones incidentales, así como las diferencias en términos de inversión de energía en la construcción de este comparativamente sencillo receptáculo funerario, sugieren fuertemente que nos hallamos en presencia de un individuo con un estatus social diferente, más bien bajo, respecto de los inhumados en las restantes estructuras que son típicas de este cementerio.

Efectivamente, no son solamente los rasgos arquitectónicos que mencionamos para la mayoría de las tumbas de La Falda, sino también la cantidad y calidad de las inclusiones funerarias, los que denotan una particular riqueza. Utilizando la clasificación de Salomon (1986) podemos separar las inclusiones encontradas en las tumbas en: a) suntuarias, y b) no suntuarias o populares. Cada una de estas clases es divisible, a su vez, según su procedencia sea local o exótica. Los objetos populares son aquellos de uso diario, habitual, por parte de la gente común. Los suntuarios, generalmente son objetos materiales que adquieren su significado y valor sociopolítico ya sea por su rareza, por su valor intrínseco, por el trabajo invertido en su confección, por el significado/valor simbólico atribuido a la materia, o por la identidad social de las personas que los utilizan (Costin 1998). Son los que confieren un mayor prestigio a su portador. Dentro de los suntuarios podemos agrupar por una parte: 1) a los elementos de lujo; 2) a los simbólicos o ceremoniales, y 3) a aquellos que están relacionados con las atribuciones de poder dentro del grupo. Una síntesis de estas consideraciones se presenta en la Tabla 2.

\section{Aspectos interpretativos}

La manifestación de estatus en las prácticas mortuorias de La Falda muestra una separación marcada en el rol social de los sexos, reflejando, a la vez, una división por género de ciertas actividades. Así, por ejemplo, sólo en enterratorios femeninos se encuentran implementos correspondientes a la actividad textil, mientras que los elementos propios de un guerrero, o ciertas herramientas tales como cinceles, hachuelas, punzones, son exclusivos de los enterratorios masculinos. Esta circunstancia coincide con la información aportada por un número importante de cronistas que describieron las sociedades andinas (Arriaga 1621; Cieza de León 1922 [1553]; Guamán Poma 1987 [1615]).

Así, los utensilios relacionados con la tejeduría (agujas, lanzaderas, husos, torteros) tienen significado de género, encontrándoselos en forma generalizada mayoritariamente en enterratorios femeninos (Arriaga 1621; Silverblatt 1987). Además, se trata de objetos populares y generalmente locales. Si bien es cierto que los hombres podían colaborar en algunas de las etapas del proceso de confección de tejidos, la labor textil era por excelencia una tarea femenina, sin importar la clase social a la que estas mujeres pertenecían. Las mujeres participaban en esta labor desde muy pequeñas y, según relata Guamán Poma (1987 [1615]), las niñas estaban encargadas de recolectar las plantas de las que se extraerían las tinturas para las telas. Según Costin (1998), desde aproximadamente los nueve años de edad hasta edades avanzadas, la principal tarea femenina era el tejido. En los hogares del común del pueblo se elaboraba la awasca, tejido liso de algodón y lana de llama, confeccionado en una trama relativamente rústica, que se utilizaba habitualmente para fabricar las vestimentas diarias de esta gente y la mayor parte de los soldados. Se la repartía tanto dentro del mismo hogar, donde se las fabricaba, como entre las otras viviendas del poblado, y entre los integrantes de las fuerzas militares.

También tejían las mujeres de la élite, y era en este tipo de unidades domésticas que se confec-

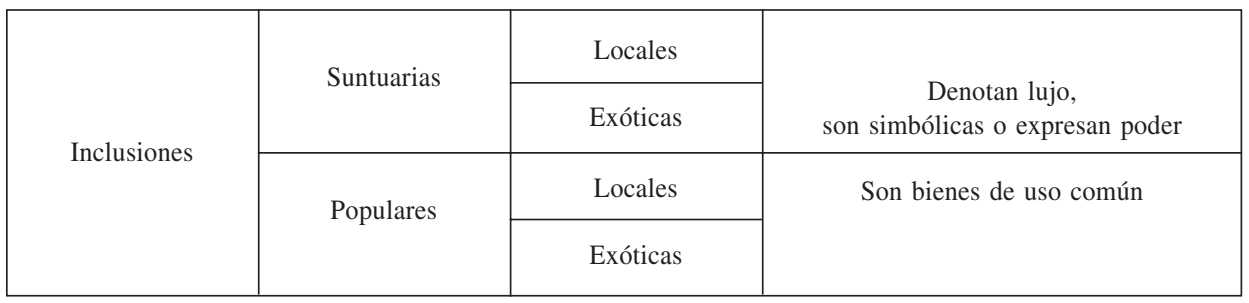

Tabla 2. Clasificación de las inclusiones funerarias según Salomon (1986). 
cionaba el cumbi, una tela decorada hecha de una lana fina, generalmente alpaca o vicuña. En su gran mayoría, el cumbi estaba confeccionado con hilos teñidos de varios colores con diseños variados. Algunos cumbi lisos tenían adornos de plumas o discos de metal (Costin 1998: 24-125). El cumbi era la tela utilizada por las clases sociales altas. Esta tela era privativa del Inka, y sólo por su voluntad podía distribuirse entre los altos funcionarios de su administración. Después de la conquista imperial, algunas élites locales fueron seleccionadas para formar parte de la burocracia inca, y a menudo sus miembros terminaban ocupando cargos de privilegio. Estas élites llegaron a formar una nueva clase de representantes administrativos dentro del Estado imperial. Tales individuos fueron, en parte, enculturados e integrados al sistema imperial, permitiéndoseles usar el tejido del Inka, como un reconocimiento y distinción respecto del cargo que ocupaban (Costin 1998: 124).

En el sitio de La Huerta (Huacalera, quebrada de Humahuaca), Debenedetti (1918) registró el hallazgo de una tumba en la que se depositaron tres individuos a los que considera "de categoría". Los mismos estaban acompañados con inclusiones muy similares a las de La Falda. Dos de los individuos conservaban vinchas de plata en sus cabezas, había también un collar de cuentas venecianas, y una muy importante cantidad de instrumentos vinculados al quehacer textil. Esta tumba ha sido interpretada como una prueba de la existencia en La Huerta de cumbicamayoc, es decir, tejedores del Inka (Raffino y Palma 1993; Palma 1998). A juzgar por estas evidencias, el individuo de la tumba 17 no sólo poseía un alto rango, sino que formaba parte de una élite de privilegio.

Como hemos mencionado, en las tumbas masculinas se han hallado cinceles y punzones confeccionados en bronce o hierro, tanto enmangados como sin enmangar; hachuelas, puntas de proyectil "cola de golondrina" (González y Pérez Gollán 1972) en hueso, arcos y astiles de madera, y también piedras de afilar. La mayoría de estos artefactos corresponden a utensilios propios de este sexo, pero serían de uso común y diario.

En cambio, los arcos de madera, presentes en las tumbas masculinas $11,16,17$ y 23 , y asociados a otros artefactos denotadores de pertenencia al grupo de guerreros, como las puntas de proyectil de hueso, que si bien son raras en la zona, también aparecieron en otros lugares de los valles y quebradas, como es el caso del sitio Rincón Chico21, valle de Santa María (Tarragó et al. 20042005), y de Cachi Adentro, en este último caso también en un contexto de tumbas en forma de bota (Tarragó 1984). Según Palma (1998), estos hallazgos parecerían ser privativos de una clase social alta.

La tumba 23 consiste en el enterratorio de un individuo masculino adolescente, de aproximadamente 16 años, con la cara y el cuerpo cubiertos por una tela muy fina, con un diseño consistente en pequeños dameros de color claro natural y negro. Según Costin (1998), los diseños en damero, de neta filiación incaica, eran utilizados por los soldados. Esta circunstancia sugiere fuertemente la adscripción de este adolescente o en todo caso de sus familiares más directos, a un grupo de élite con nexos militares (Guamán Poma 1987 [1615]). Es notable el paralelismo existente entre las restantes inclusiones de esta tumba y aquellas que corresponden a tumbas que son propias de varones adultos. En la tumba 23, el menor tamaño tanto relativo como absoluto de tales inclusiones (palita de madera, arquito, puntas pequeñas, entre otros) refuerza los aspectos etarios del inhumado y señalan que este inmaduro no había alcanzado todavía la edad requerida para participar plenamente de las actividades propias de una clase militar (Guamán Poma 1987 [1615]: 188). En nuestra opinión, se trataría del entierro de un inmaduro que si bien socialmente debió haber visto materializado su rito de pasaje hacia la adultez, los significados de las inclusiones funerarias que lo acompañan responden más a una intencionalidad vinculada con las funciones propias de la pertenencia ocupacional de sus deudos más directos.

El contexto ceremonial del conjunto recuperado en La Falda está también representado por elementos del complejo de alucinógenos. Se trata de tubos para aspiración de polvos realizados sobre huesos de ave ensamblados, tabletas simples, sin decoración, pequeñas bolas de llijta, y pequeños morteritos asociados. Asimismo, hay bolsitas de cuero conteniendo en su interior polvos $\mathrm{y} / \mathrm{o}$ pigmentos (cobre y mica), y vasos libatorios en la forma de keros de madera, que siempre van de a pares, en estrecha proximidad espacial entre sí. Los tubos, confeccionados en hueso largo de ave, y cubiertos con corteza, la tableta y las bolsas de 
pigmentos, se encontraron en tumbas de varones adultos. Los keros se encuentran en dos tumbas femeninas y una masculina.

Los objetos suntuarios, ya sean locales o exóticos, se han encontrado en su mayoría en tumbas masculinas. Así, en la tumba 21, correspondiente a un individuo de alrededor de 30 años de edad, se encontró un disco de bronce de unos $7 \mathrm{~cm}$ de diámetro. Según Rowe (1946), estos objetos, que solían llevarse colgados en el pecho o sobre la frente, eran otorgados por el Inka a aquellos soldados que se destacaban en la acción bélica, como premio a la bravura. Debemos señalar que este mismo individuo portaba en su frente fragmentos de una cinta de plata, a modo vincha y en el brazo izquierdo otro fragmento a modo de brazalete. Sobre su pecho se encontró una concha de bivalvo marino. El joven varón de la tumba 23 también tenía un brazalete de sección troncocónica, pero esta vez de bronce y muy bien definido en sus dimensiones. También se encontraron junto al cuerpo láminas del mismo material, que probablemente hayan estado adosadas a la vestimenta.

En las tumbas femeninas 18 y 19 , y en la masculina 17, se han encontrado vinchas de plata rodeando las frentes de sus poseedores. Cabe destacar que en este último enterratorio, caracterizado por su excelente preservación, y donde había sido depositado un individuo masculino de unos 20 años, se conservaba todavía en relativamente buen estado el tocado de lana roja con algunas hebras azules que había adornado su cabeza, y que estaba rodeado y sujeto con una vincha de plata. Esta tenía la forma de una cinta de aproximadamente $2 \mathrm{~cm}$ de ancho que daba seis vueltas alrededor de la cabeza. Como mencionamos, también conservaba parte de su vestimenta. La presencia de prendas de origen foráneo (telas de origen europeo), formando parte de la vestimenta del individuo de la tumba 17, plantea también la utilización de elementos de prestigio y refuerza nuestras apreciaciones anteriores. Según Palermo (2000) la utilización de vestimentas europeas entre personajes indígenas de importancia no parece haber sido un hecho infrecuente.

Otro elemento suntuario de origen exótico que aparece profusamente en muchas de las tumbas de La Falda, asociándose indistintamente respecto del sexo, son las cuentas de cristal o "venecianas". Elaboradas en Europa (primero en Italia y luego también en España), fueron objeto de una muy amplia distribución por parte de los europeos en diversas regiones del mundo. Al parecer, llegaron por vez primera a América con los viajes de Colón (Smith y Good 1982). Se las ha encontrado desde el sur de Canadá hasta Patagonia (Gómez Otero 2003). Las que aparecen en La Falda corresponden a modelos fabricados en el siglo XVI (Smith y Good 1982). Según mencionamos, las cuentas venecianas utilizadas por el grupo que generó este cementerio no se usaron exclusivamente para formar parte de collares, sino que, según parece, y conforme a las condiciones de hallazgo, recolección y registro, también podrían haber estado cosidas a la vestimenta. En efecto, en la tumba 18 (entierro femenino doble) en toda la extensión de uno de los esqueletos se encontró una importante cantidad de cuentas venecianas de tamaño muy pequeño, lo cual sugiere su posible costura en algunas prendas, a modo de mostacillas.

La presencia de elementos tanto suntuarios como no suntuarios de origen exótico entre los bienes registrados en las tumbas de La Falda, nos permite suponer la existencia de redes dinámicas de conexión y de intercambio tanto a corta como a larga distancia de dichos bienes. En La Falda, la evidencia de este intercambio y estas interconexiones se corresponderían con la época incaica, llegando a extenderse hasta los primeros contactos e intercambios con los españoles. Recordemos que si bien los incas tuvieron un período de dominación relativamente corto en la actual Argentina, el cual aparentemente se materializó entre 1480 y la caída de Atahualpa en manos de los españoles en 1532, el mismo alcanzó una muy amplia extensión territorial. Durante ese lapso el orden sociopolítico en el Noroeste Argentino sufrió cambios significativos (Nielsen 2001), ya que entre otros aspectos de importancia, se trasladaron a las tierras conquistadas las instituciones y hasta pueblos enteros, a fin de lograr sus propósitos de extensión fronteriza, dominación, y organización estatal.

En el caso particular de la quebrada de Humahuaca, el impacto producido por la penetración territorial y cultural de los conquistadores incaicos se manifiesta en la presencia de cambios arquitectónicos, y también en la cultura material y no material. No sólo se modificaron algunos asentamientos previos levantándose en ellos cons- 
trucciones de neto patrón incaico, sino que también se establecieron nuevos asentamientos y se produjo el abandono de otros (Nielsen 2001). En la dinámica de esta penetración estaba contemplada y bien planificada la instalación de una nueva red de tributarios del Inka. Los mismos eran ocupados en labores agrícolas, mineras, textiles y lapidarias, entre otras. El control de estas actividades era ejercido por funcionarios administrativos nombrados por el mismo Inka. Entre ellos podían contarse tanto los mismos señores y/o líderes de los grupos étnicos locales a medida que eran incorporados al sistema, como los señores y/o líderes de grupos étnicos no locales, que eran trasladados a tal efecto desde otras regiones. En lo que respecta a las posibles relaciones de filiación (procedencia local o foránea, o vínculos estrechos o no de parentesco) de los individuos recuperados en La Falda, se destaca una característica expresión ósea de carácter congénito, que se presenta en forma recurrente en tres de las mujeres depositadas en tumbas botiformes. Se trata de la malformación conocida como espina bífida, en la cual la lámina de uno o más arcos neurales no alcanza a fusionarse en la línea media (Martini 2001), es la más común de todas las anomalías espinales congénitas y se localiza por lo general en la porción lumbo-sacra (Aufderheide y Rodríguez-Martin 1998). Investigaciones clínicas recientes han indicado un fuerte componente genético en la incidencia de esta dolencia (Scheuer y Black 2000); dichos hallazgos sugieren que en La Falda la presencia de espina bífida podría ser la expresión de un cierto grado de endogamia.

Este estado de cosas se mantuvo hasta la irrupción y subsecuente conquista europea en el escenario andino. En la quebrada de Humahuaca se produce una desestructuración política, como consecuencia de la caída del Imperio Incaico a manos de los europeos. Al poco tiempo de sucedido esto, comienzan a tomar fuerza e independencia los señores étnicos locales (Palomeque 2000: 105). El control efectivo de la región por parte de los españoles no se pudo materializar, sino hacia fines del siglo XVI, que es cuando se produce el apresamiento del curaca quebradeño Viltipoco, a manos de los hombres de Argañaraz. Pero en los años que median entre la caída del Inka Atahualpa y la del cacique Viltipoco, había comenzado ya el intercambio entre los conquistadores europeos y las poblaciones indígenas de la quebrada. Notablemente, y a pesar de que el gobernador del
Tucumán justifica la captura del curaca quebradeño haciéndolo responsable de la organización de una sublevación general, desconociendo pactos anteriores, Viltipoco había acordado poco antes su sujeción a la Audiencia de Charcas, y hay pruebas de que efectivamente tributaba a ella (Palomeque 2000).

\section{Conclusiones}

Este trabajo se ha basado fundamentalmente en el tratamiento de la evidencia recuperada en aquellas unidades inhumatorias que se encontraron intactas dentro del Cementerio de La Falda. Los resultados, las interpretaciones y las conclusiones que de él se derivan deben remitirse fundamentalmente a dicha evidencia sistemáticamente recuperada. Queda abierta la posibilidad de que, de producirse otros hallazgos similares y de contarse con una casuística mayor, la interpretación de los diferentes ítems e inclusiones funerarias permita la incorporación de nuevos atributos a la lista de rasgos y características que aquí estipulamos y reconocemos como de valor diagnóstico.

Una serie de consideraciones y el análisis particularizado de cierto tipo de atributos tales como el tipo de tumba que, como dijimos, en los Andes generalmente se asocia a grupos de élite, como la cantidad, variedad y calidad de las inclusiones funerarias encontradas en este cementerio, sugieren fuertemente la pertenencia del grupo representado en La Falda a un estrato social alto.

Efectivamente, tanto los varones como las mujeres de esta área de entierro colectivo poseen, además de los atributos identificatorios propios de su sexo, una serie de elementos que son propios de su clase y que permiten explorar significados dentro de un escenario tanto prehistórico como histórico del devenir sociocultural de las sociedades quebradeñas, con particular énfasis respecto de lo acontecido en la localidad de Tilcara.

La mayor parte de los enterratorios masculinos están acompañados por elementos propios de su persona social donde se destaca su condición de guerrero. Los entierros femeninos están fuertemente acompañados de objetos muchas veces decorados que talvez podrían estar asociados al quehacer textil elaborado. En los Andes prehispánicos los textiles poseían valor económico, político y ritual. En la misma línea de pensamiento, es posi- 
ble entonces proponer una situación para la interpretación de la actividad por excelencia desarrollada por las mujeres de la clase alta de La Falda equivalente a aquella postulada para La Huerta (Raffino y Palma 1993).

En las sociedades tradicionales, generalmente la noción de distancia geográfica está estrechamente relacionada con lo cosmológico religioso. Ello involucra un contraste marcado entre lo local (la sociedad) y lo exterior (espacio cosmológico) (Helms 1993). Entre ambos dominios tiene lugar la ocurrencia de una distancia cosmológica. De este modo, la presencia de bienes de intercambio con otros grupos y regiones puede interpretarse desde diversas perspectivas: a) en términos económicos como, por ejemplo, las relaciones económicas verticales o de complementariedad (Murra 1972); b) en términos políticos, como medio de legitimación de la autoridad política (Peregrine 1991), y c) en términos ideológicos, en tanto indicadores de una actividad de índole político ideológica (Helms 1993).

Los grupos destacados dentro de una sociedad buscan obtener contactos e incorporar conocimientos esotéricos provenientes de dominios cosmológicamente diferentes respecto la sociedad local (Helms 1993). En las sociedades andinas, especialmente en el mundo incaico, los individuos con mayor influencia provenían de los ámbitos político, militar y religioso. Los señores nativos representaban en esa época el máximo poder político a nivel local, y estaban encargados de controlar los dominios ubicados en la distancia geográfica (Salomon 1986). Esta posibilidad de contacto o adquisición de bienes procedentes de ámbitos ubicados a gran distancia respecto de la sociedad local formaba parte del conocimiento esotérico controlado por especialistas político-religiosos.

Todas las evidencias anteriormente mencionadas sugieren fuertemente que el conjunto de La Falda representaría a un grupo de élite, posiblemente constituido por miembros de estados vecinos a los cuales se les había otorgado estatus oficial. Probablemente corresponderían a la categoría de "incas de privilegio" (Zuidema 1982). Los "incas de privilegio" constituían un grupo asociado u "honorario" (Cahill 2005), elevado a esa condición ya fuera por razones de gobierno o por haber desempeñado funciones a favor del Estado. Bauer (1992) considera que los "incas de privilegio" jugaron roles importantes en la organización del Imperio, y ocuparon posiciones administrativas destacadas en los nuevos territorios anexados.

La presencia de la misma manifestación de carácter congénito en los sacros de tres mujeres del grupo, enterradas en proximidad unas de otras, permite inferir la existencia de relaciones de parentesco más o menos cercano entre estos individuos. La anciana depositada en la única tumba con modalidad constructiva y con inclusiones diferentes a la norma de este cementerio podría interpretarse como el resultado de la incorporación de un representante de un estrato social de menor jerarquía dentro del espacio mortuorio de la élite.

El patrón de las tumbas, notablemente diferente al patrón tardío generalizado para el área, sugiere, asimismo, que el Cementerio de La Falda talvez albergó a un grupo de mitimaes o colonizadores trasplantados por el Estado Inca. El estatus de estos colonizadores se asociaba con grupos de especialistas económicos, tales como tejedores, ceramistas, artesanos en metal, y preparadores de chicha (Espinoza Soriano 1987). En el caso que nos ocupa, podría muy bien tratarse de funcionarios administrativos, guerreros y cumbicamayoc.

Agradecimientos Deseo agradecer muy especialmente a los dos revisores anónimos de esta revista, quienes, si bien desde una primera instancia consideraron que el trabajo era publicable, han contribuido grandemente con sus sugerencias y comentarios críticos a mejorar el manuscrito original. Cualquier error u omisión conceptual son, obviamente, de mi exclusiva responsabilidad. 


\section{REFERENCIAS CITADAS}

ARRIAGA, P. P. J., 1621. Extirpación de la idolatría del Pirú. Gerónimo de Contreras, Lima.

AUFDERHEIDE, A. y C. RODRIGUEZ-MARTIN, 1998. The Cambridge Encyclopedia of human paleopathology, Cambridge University Press, Cambridge.

BARBOZA, M. C., O. MENDONÇA y M. A. BORDACH, 2001. Estimadores anatómicos y la edad de la muerte: Resultados de su aplicación a los materiales del Sitio SJTil 43 (Tilcara, Jujuy) Revista de la Asociación Argentina de Antropología Biológica 3 (2): 49-63.

-2002a. Contexto arqueológico y biología esqueletal humana. Actas del XIII Congreso Nacional de Arqueología Argentina T 2, pp. 339-345.

2002b. Estudio de marcadores biológicos y culturales del sexo en un conjunto hispano-indígena de Tilcara (sitio SJTil 43). Revista de la Asociación Argentina de Antropología Biológica 4 (1): 87-111.

2003-2004. Expresión del dimorfismo sexual mandibular de esqueletos del sitio SJTil 43 (Tilcara, Jujuy). Revista de la Asociación Argentina de Antropología Biológica 5 (2): 63-74.

BAUER, B. S., 1992. The development of the Inca State, University of Texas Press, Austin.

BORDACH, M. A., O. MENDONÇA, M. RUIZ y M. E. ALBECK, 1998. El "Joven Señor" de La Falda: Indicadores de una persona social en el Tilcara hispanoindígena. En Los desarrollos locales y sus territorios, M. B. Cremonte, (Ed.), pp. 199-208. Universidad Nacional de Jujuy, San Salvador de Jujuy.

BORDACH, M. A. y O. MENDONÇA, 2001. The young Lord of La Falda, markers of a social persona in early contact Tilcara. En Mortuary practices and ritual associations. Shamanic elements in prehistoric funerary contexts in South America, E. Currie y J. E. Staller (Eds. ), pp. 129135. British Archaeological Reports (BAR), Cambridge.

BROWN, J. A., 1971. The dimensions of status in the burials at Spiro. En Approaches to the social dimensions of mortuary practices, J. A. Brown (Ed.), pp. 92-112. Society for American Archaeology, Washington D. C.

CADAVID CAMARGO, G. y H. ORDOÑEZ, 1989. Arqueología de salvamento de la vereda de Tajumbina, Municipio de la Cruz (Nariño). Fundación de Investigaciones Arqueológicas Nacionales del Banco de la República, Bogotá.

CAHILL, D. 2005. El rostro del inca perdido. La Virgen de Loreto, Tocay Cápac y los ayamarcas en el Cuzco colonial. Documento de Trabajo 146. Serie Historia, 28, Instituto de Estudios Peruanos, Lima.

CHAPMAN, R. W., 1977. Burial practices: An area of mutual interest. En Archaeology and anthropology: Areas of mutual interest, M. Spriggs (Ed.), pp. 19-33. British Archaeological Reports (BAR), Oxford.

CHAPMAN, R. W. y K. RANDSBORG, 1981. Perspectives on the archaeology of death. En The archaeology of death, R. W. Chapman, I. Kinnes y K. Randsborg (Eds. ), pp. 1-24. Cambridge University Press, Cambridge.

CHESSON, M. S., 2001. Social memory, identity, and death: An introduction. En Social memory, identity, and death: Anthropological perspectives on mortuary rituals, M. S. Chesson (Ed.), pp. 1-10. American Anthropological Association, Arlington.

CIEZA DE LEON, P., 1922 [1553]. La crónica del Perú. Calpe, Madrid.

COSTIN, C. L., 1998. Housewives, chosen women, skilled men: Cloth production and social identity in the Late Prehispanic Andes. En Craft and social identity, C. L. Costin y R. P. Wright (Eds.), pp. 123-141. American Anthropological Association, Arlington.

DEBENEDETTI, S., 1918. La XIV expedición arqueológica de la Facultad de Filosofía y Letras. Physis 4: 196-207.

DOYON, L. G., 2002. Conduits of ancestry: Interpretation of the geography, geology and seasonality of North Andean shaft tombs. En The space and place of death, $\mathrm{H}$. Silverman y D. B. Small (Eds.), pp. 79-95. American Anthropological Association, Arlington.

DRENNAN, R., 1995. Mortuary practices in the Alto Magdalena: The social context of the "San Agustín Culture". En Tombs for the living: Andean mortuary practices, $\mathrm{T}$. Dillehay (Ed.), pp. 79-110. Dumbarton Oaks, Washington D. C.

DUQUE GOMEZ, L., 1999. Notas sobre la investigación arqueológica en Colombia en la década de los 90 . Boletín Cultural y Bibliográfico del Banco de la República 36: $50-51$

ESPINOZA SORIANO, W., 1987. Migraciones internas en el Reino Colla: Tejedores, plumeros y alfareros del Estado Imperial Inca. Chungara 19: 243-263.

GOLDSTEIN, P., 1989. Omo, a Tiwanaku provincial center in Moquegua, Perú. Ph. D. Dissertation. The University of Chicago, Chicago.

GOMEZ OTERO, J., 2003. Movilidad y contactos en la costa centro-norte de Patagonia argentina en tiempos pre y posthispánicos. En Las fronteras hispano-criollas del mundo indígena latinoamericano en los siglos XVIII-XIX. Un estudio comparativo, R. Mandrini y C. Paz (Eds.), pp. 287-312. Universidad Nacional del Centro de la Provincia de Buenos Aires, Universidad Nacional del Comahue, Universidad Nacional del Sur, Neuquén.

GOODENOUGH, W. H., 1965. Rethinking "status" and "role": Toward a general model of the cultural organization of social relationships. En The relevance of 
models for social anthropology, M. Banton (Ed.), pp. 1-24. Tavistock, Londres.

GONZALEZ, A. R. y J. A. PEREZ GOLLAN, 1972. Argentina indígena. Vísperas de la conquista. Paidós, Buenos Aires.

GROSSO, M., O. MENDONÇA, M. A. BORDACH y L. B. DALERBA, 1998. Restos vegetales con valor alimenticio y/o culinario del sitio SJTil 43. Tratamiento por flotación. En Los desarrollos locales y sus territorios, M. B. Cremonte (Ed.), pp. 209-222. Universidad Nacional de Jujuy, San Salvador de Jujuy.

GROSSO, M., O. MENDONÇA, M. A. BORDACH y M. A. CAPPONE, 2001-2002. Identificación de semillas en el cementerio de La Falda (SJTil 43). Flotación, Segunda Parte. Actas del XIII Congreso Nacional de Arqueología Argentina, T 2, pp. 455-465.

GUAMAN POMA DE AYALA, F., 1987 [1615]. Nueva crónica y buen gobierno. Historia 16, Madrid.

HÄRKE, H., 1997. The nature of burial data. En Burial and society: The chronological and social analysis of archaeological burial data, C. Kjeld Jensen y K. Høilund Nielsen (Eds.), pp. 19-28. Aarhus University Press, Aarhus.

HELMS, M. W., 1993. Craft and the kingly ideal. University of Texas Press, Austin.

HODDER, I., 1982. Symbols in action: Ethnoarchaeological studies of material culture. Cambridge University Press, Cambridge.

KAULICKE, P., 1997. La muerte en el Antiguo Perú. Contextos y conceptos funerarios: Una introducción. Boletín de Arqueología de la Pontificia Universidad Católica del Perú 1 (1): 7-54.

KRAPOVICKAS, P., 1977. Arqueología de Cerro Colorado (Departamento Yavi, Provincia de Jujuy, República Argentina). En Obra del centenario del Museo de La Plata, pp. 123-148, Museo de La Plata, La Plata.

LINTON, R., 1936. The study of man. Appleton-Century Company, Nueva York.

MARTINI, F. H., 2001. Fundamentals of anatomy and physiology. Prentice Hall, Nueva Jersey.

MAURER TRINKAUS, K., 1995. Mortuary behavior, labor organization, and social rank. En Regional approaches to mortuary analysis, L. A. Beck (Ed.), pp. 53-75. Plenum Press, Nueva York.

MENDONÇA, O. y M. A. BORDACH, 2001 Ritual and symbolism in mortuary behavior. Bio-cultural, chronological and regional facts in Northwestern Argentina. En Mortuary practices and ritual associations. Shamanic elements in prehistoric funerary contexts in South America, E. Currie y J. E. Staller (Eds. ), pp. 137-143. British Archaeological Reports (BAR), Oxford.
MENDONÇA, O., M. A. BORDACH, M. E. ALBECK y M. RUIZ, 1997. Collares de vidrio y ollas de barro: Comportamiento ante la muerte en el Tilcara hispano-indígena inicial (Jujuy, Argentina). Cuadernos 9: 175-202.

MENDONÇA, O., M. A. BORDACH y M. GROSSO, 2003. Ocupación territorial y control e intercambio en el Período Hispano-indígena. Estudio comparado de los cementerios R Ch21 (Catamarca) y SJTil 43 (Jujuy). Cuadernos 20: 221-237.

MOORE, J, y E. SCOTT, (Eds), 1997. Invisible people and processes: Writing gender and childhood into European archaeology. Leicester University Press, Leicester.

MURRA, J., 1972. El control vertical de un máximo de pisos ecológicos en la economía de las sociedades andinas. En Visita de la Provincia de León de Huanuco en 1562, J. Murra (Ed.), pp. 427-476, Universidad Nacional Hermilio Valdizán, Huanuco.

NIELSEN, A., 2001. Evolución social en quebrada de Humahuaca (700-1536 DC). En Historia argentina prehispánica, E. Berberián y A. Nielsen (Eds.), pp. 171264. Editorial Brujas, Córdoba.

NIEMEYER, H., 1986. La ocupación incaica en la cuenca alta del río Copiapó. Comechingonia vol. Homenaje, $45^{\circ}$ Congreso Internacional de Americanistas, Córdoba.

O'SHEA, J., 1984. Mortuary variability: An archaeological investigation. Academic Press, Nueva York.

PALMA, J. R., 1998. Curacas y señores. Una visión de la sociedad política prehispánica en la quebrada de Humahuaca. Serie Monográfica. Instituto Interdisciplinario Tilcara, Tilcara.

PADER, E., 1982. Symbolism, social relations and the interpretation of mortuary remains. British Arachaeological Reports (BAR), Oxford.

PALERMO, M. A., 2000. A través de la frontera. Economía y sociedad indígenas desde el tiempo colonial hasta el siglo XIX. En Los pueblos originarios y la conquista. Nueva Historia Argentina, M. Tarragó (Ed.), pp. 341-382. Editorial Sudamericana, Buenos Aires.

PALOMEQUE, S., 2000. El mundo indígena, siglos XVIXVIII. En La sociedad colonial. Nueva Historia Argentina, E. Tandeter (Ed.), pp. 87-143. Editorial Sudamericana, Buenos Aires.

PARKER PEARSON, M., 1982. Mortuary practices, society and ideology: An ethnoarchaeological study. En Symbolic and structural archaeology, I. Hodder (Ed.), pp. 99-113. Cambridge University Press, Cambridge.

2000. The archaeology of death and nurial. Texas A \& M University Press, Somerset.

PEREGRINE, P., Political aspects of craft especialization. World Archaeology 23 (1): 1-11. 
RAFFINO, R. y J. R. PALMA, 1993. Los artefactos. En Inka. Arqueología, historia y urbanismo del altiplano andino, R. Raffino (Ed.), pp 93-129. Corregidor, Buenos Aires.

ROWE, J., 1946. Inca culture at the time of the Spanish Conquest. En Handbook of South American Indians, J. Steward (Ed.), pp. 183-330. Bureau of American Ethnology, Washington D. C.

SALOMON, F. L., 1986. Native lords of Quito in the age of the incas: The political economy of North Andean chiefdoms. Cambridge University Press, Cambridge.

SAXE, A, 1970. Social dimensions of mortuary practices. $\mathrm{Ph}$. D. Dissertation. The University of Michigan, University Microfilms, Ann Arbor.

SCHEUER, L. y S. BLACK, 2000. Developmental juvenile osteology. Academic Press, San Diego.

SILVERBLATT, I., 1987. Moon, sun and witches. Princeton University Press, Princeton.
SMITH, M. T. y M. E. GOOD, 1982. Early Seventeenth Century glass beads in the Spanish colonial trade. Cottonlandia Museum Publications, Greenwood.

TAINTER, J. A., 1978. Mortuary practices and the study of prehistoric social systems. En Advances in archaeological method and theory, vol 1, M. Schiffer (Ed.), pp. 105-141. Academic Press, Nueva York.

TARRAGO, M., 1984. El contacto hispano-indígena: La provincia de Chicoana. Runa XIV: 143-185.

TARRAGO, M., M. A. BORDACH y O. MENDONÇA, 20042005. El Cementerio de Rincón Chico 21. Santa María (Catamarca). En IV Jornadas de Arqueología y Etnohistoria del Centro-Oeste de la Argentina y Seminario de Etnohistoria, vol. 2, M. Bechis (Ed.), vol. II: 31-40. Facultad de Ciencias Humanas, Universidad Nacional de Río Cuarto, Río Cuarto.

ZUIDEMA, T., 1982. Bureaucracy and systemic knowledge in Andean civilizations. En The Inca and Aztec States, 1400-1800, anthropology and history, G. A. Collier, R. I. Rosaldo y J. D. Wirth (Eds.), pp. 419-458. Academic Press, Nueva York. 